\title{
Microwave-assisted synthesis of peptidyl phosphorus ylides
}

\author{
Alan R. Katritzky,* Adam S. Vincek, and Kazuyuki Suzuki \\ Center for Heterocyclic Compounds, Department of Chemistry, \\ University of Florida, Gainesville, FL 32611-7200, USA \\ E-mail: katritzky@,chem.ufl.edu \\ Dedicated to Professor Fišera on his $60^{\text {th }}$ anniversary \\ (received 11 Feb 05; accepted 18 Mar 05; published on the web 26 Mar 05)
}

\begin{abstract}
Chiral $N$-Boc- and Cbz-protected ( $\alpha$-aminoacyl)benzotriazoles $\mathbf{6 a}-\mathbf{g}$ microwaved for 10 minutes with ethyl (triphenylphosphoranylidene)acetate 7 produce chiral phosphorus ylides $8 \mathbf{a}-\mathbf{g}$ in 65-90\% yield. Reactions of diastereomeric chiral phosphorus ylides $(\mathbf{1 5}, \mathbf{1 6})$ demonstrated preservation of chirality.
\end{abstract}

Keywords: Phosphorus ylide, $N$-( $\alpha$-aminoacyl)benzotriazoles, microwave, benzotriazole methodology

\section{Introduction}

Peptidyl phosphorus ylides have attracted considerable attention as important intermediates for the preparation of peptidyl $\alpha$-keto esters and $\alpha$-keto amides, which are potential inhibitors for proteolytic enzymes $^{1 \mathrm{a}, 1 \mathrm{~b}}$ and leukotriene $\mathrm{A}_{4}$ hydrolase. ${ }^{1 \mathrm{c}}$ Acyl phosphorus ylides $\mathbf{1}$ have been used for (i) synthesis of olefins 2, (ii) preparation of alkynes $\mathbf{3}$ by flash vacuum pyrolysis (FVP), ${ }^{2}$ (iii) formation of tricarbonyl compounds 4 by oxidation with $\mathrm{O}_{3}{ }^{3 \mathrm{a}}$ or oxone ${ }^{\circledR},{ }^{3 \mathrm{~b}}$ and (iv) direct reduction with Al-Hg to obtain $\beta$-keto esters $\mathbf{5}^{4}$

Diverse acyl phosphorus ylides $\mathbf{1}$ are readily prepared by reactions of phosphorus ylides with acyl chlorides in the presence of a base. ${ }^{3,5}$ Peptidyl phosphorus ylides are commonly prepared in a one-pot sequence by activation of the carboxyl group in $\mathrm{N}$-protected amino acids with $\mathrm{CDI}^{6}$ or 1-(3-dimethylaminopropyl)-3-ethylcarbodiimide hydrochloride (EDCl)/DMAP, ${ }^{3,5 b, 7}$ but the bases used are a potential source of low yields and problems with other functional groups. Thus, the development of a new method for the acylation of amino acid-derived phosphorus ylides without the use of base is desirable. 


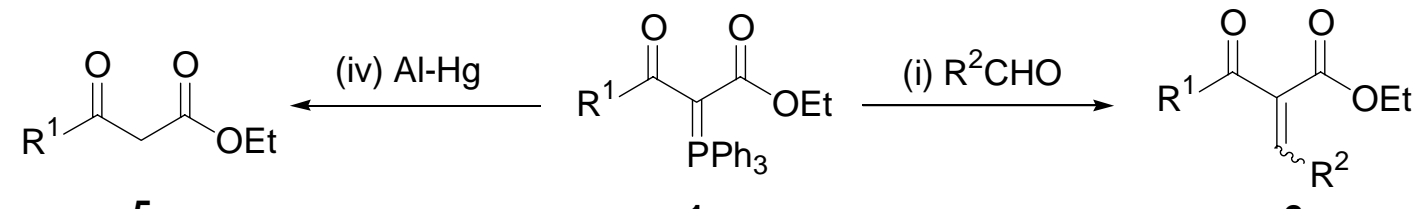<smiles>[R]C(=O)C(=O)C(=O)OCC</smiles>

4

1

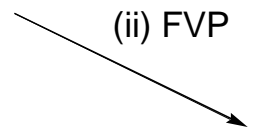

2

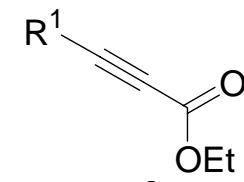

3

\section{Scheme 1}

$N$-Protected ( $\alpha$-aminoacyl)benzotriazoles are effective chiral acylating reagents for the convenient preparation of amino amides, ${ }^{8 \mathrm{a}}$ amino sulfonamides, ${ }^{8 \mathrm{~b}}$ amino thiol esters, ${ }^{8 \mathrm{c}}$ small peptides carrying side-chains in the first component with alkyl groups, ${ }^{8 \mathrm{~d}}$ small peptides with multi-functional groups, ${ }^{8 \mathrm{e}}$ and amino ketones. ${ }^{8 \mathrm{f}}$ We now demonstrate acylation of phosphorus ylides with $N$-protected (aminoacyl)benzotriazoles under microwave irradiation utilizing a single mode cavity synthesizer, ${ }^{8 \mathrm{~g}, 8 \mathrm{~h}}$ which helps to ensure reproducibility and safety. Microwave heating is also an effective technique for promoting a variety of reactions and shortening reaction times while reducing pollution.

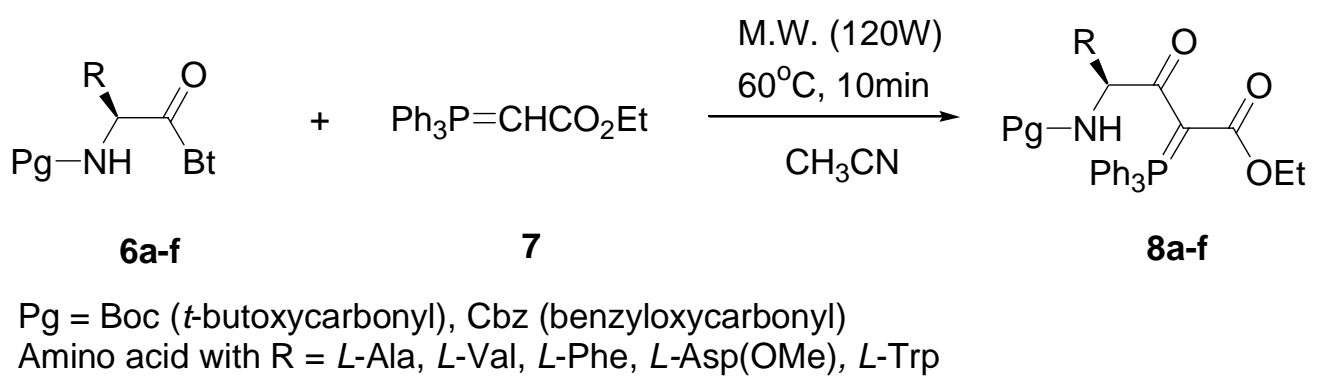

\section{Scheme 2}

\section{Results and Discussion}

\section{Preparation of $\mathrm{N}$-protected ( $\alpha$-aminoacyl)benzotriazoles}

The starting $N$-(Boc- $\alpha$-aminoacyl)benzotriazole $\mathbf{6 a},{ }^{8 \mathrm{a}} N$-(Cbz- $\alpha$-aminoacyl)benzotriazoles $\mathbf{6 b}-\mathbf{f}$ ( $L$-configuration), 6g ( $D$-configuration), and 9a-c (non-chiral) were prepared in $72-95 \%$ yields from the corresponding N-protected amino acids following recently developed procedures. ${ }^{8 \mathrm{~d}, 8 \mathrm{e}}$ Compound 6e, 6g, and 9a-c are novel, and characterized by NMR, microanalysis, and optical rotation. 


\section{Preparation of peptidyl phosphorus ylides $8 \mathbf{a}-\mathbf{g}$}

Microwave reactions were carried out in a standard $50 \mathrm{~mL}$ round bottom flask under controlled, safe and reproducible conditions. The single mode microwave irradiation was used at a fixed temperature and irradiation power, which automatically maintained the temperature.

Optimization of the reaction conditions was investigated using the reaction of Cbz- $L-\mathrm{Ala}-\mathrm{Bt}$ (6b) with 7 in $\mathrm{CH}_{2} \mathrm{Cl}_{2}, \mathrm{CH}_{3} \mathrm{CN}$, or toluene as choices of solvent with different combinations of temperature, time and irradiation power using a round bottom flask as well as a sealed heavywalled Pyrex tube. The optimized reaction of $\mathbf{6 b}$ with 7 at $60{ }^{\circ} \mathrm{C}(120 \mathrm{~W})$ in $\mathrm{CH}_{3} \mathrm{CN}$ for 10 min utilizing a round bottom flask gave the desired product $\mathbf{8 b}$ in $88 \%$ yield in pure form after waterwash work-up. By contrast, reactions performed in $\mathrm{CH}_{2} \mathrm{Cl}_{2}$ at $36{ }^{\circ} \mathrm{C}$ and $70 \mathrm{~W}$ irradiation power gave no detectable $\mathbf{8 b}$ within 30 minutes. In toluene, at $110{ }^{\circ} \mathrm{C}$ with $200 \mathrm{~W}$, signals for $\mathbf{8 b}$ were observed in the ${ }^{1} \mathrm{H}$ NMR spectrum along with signals of by-products, and the yield of $\mathbf{8 b}$ was estimated as $<30 \%$. For comparison, when thermal reactions of $\mathbf{6 b}$ and $\mathbf{6 d}$ with $\mathbf{7}$ in refluxing $\mathrm{CH}_{3} \mathrm{CN}$ were carried out, 12 hours was required to give the corresponding products $\mathbf{8 b}$ and $\mathbf{8 d}$ in $86,79 \%$ yield, respectively. Although these yields are satisfactory, the microwave protocol is beneficial for significantly shortening the reaction time and reducing the amount of solvent required. The above optimized microwave reaction condition $\left(60{ }^{\circ} \mathrm{C}, 120 \mathrm{~W}, \mathrm{CH}_{3} \mathrm{CN}, 10 \mathrm{~min}\right.$. $)$ was applied to the preparation of peptidyl phosphorus ylides $\mathbf{8 a}-\mathbf{g}$ (Table 1). The results exhibited fast and clean microwave acylation, and use of N-protected $(\alpha$ aminoacyl)benzotriazoles $\mathbf{6 a}-\mathbf{g}$ avoided the use of base.

Compound 8 a was previously prepared in 54\% yield by reaction of the corresponding Bocprotected $N$-carboxyanhydride with $7 .^{10}$ Althought this method offers reactions without preactivation of $\mathrm{N}$-protected amino acids, several steps are required to prepare the starting $\mathrm{N}$ carboxyanhydride. Direct couplings of Cbz-Ala-OH and Cbz-Val-OH with 7 were carried out in the presence of EDCl/DMAP to produce compound $\mathbf{8 b}, \mathbf{8 c}$ in $46 \%$ and $45 \%$ yield, respectively. ${ }^{2 b}$ The preparation of 12 ylides by this method produced average of yields $47 \%$. By our method, compound $\mathbf{8 a , b , c}$ were obtained in 64,88 , and $88 \%$ yield, respectively providing higher yields over the yields using the previously reported methods.

Unfortunately, attempts failed to produce the corresponding acyl phosphorus ylides from the $N$-(Cbz- $\alpha$-aminoacyl)benzotriazole prepared from Cbz-glutamine (Cbz-Glu-Bt ${ }^{8 \mathrm{e}}$ ), instead hydrolysis of Cbz-Glu-Bt regenerated Cbz-glutamine. In case of Fmoc-Ala-Bt, a reaction gave a complex mixture from which Fmoc-Bt was isolated by column chromatography $\left(\mathrm{SiO}_{2}\right)$. The formation of Fmoc-Bt could be explained by a nucleophilic substitution with the benzotriazole anion, generated by the coupling reaction, at the carbonyl carbon in the Fmoc group. 
Table 1. Preparation of chiral phosphorus ylides

\begin{tabular}{cccccc}
\hline Product & Amino acid & $\mathrm{Pg}$ & $\mathrm{R}$ & Yield $^{a}(\%)$ & Lit. yield (\%) \\
\hline $\mathbf{8 a}$ & $L$-Ala & $\mathrm{Boc}$ & $\mathrm{CH}_{3}$ & 65 & $54^{c}$ \\
$\mathbf{8 b}$ & $L$-Ala & $\mathrm{Z}$ & $\mathrm{CH}_{3}$ & $88(86)$ & $46^{d}$ \\
$\mathbf{8 c}$ & $L$-Val & $\mathrm{Z}$ & $\mathrm{CH}_{3}\left(\mathrm{CH}_{3}\right)_{2}$ & $88^{c}$ & $45^{d}$ \\
$\mathbf{8 d}$ & $L$-Phe & $\mathrm{Z}$ & $\mathrm{CH}_{2} \mathrm{C}_{6} \mathrm{H}_{5}$ & $89(79)^{b}$ & - \\
$\mathbf{8 e}$ & $L$-Asp(OMe) & $\mathrm{Z}$ & $\mathrm{CH}_{2} \mathrm{CO}_{2} \mathrm{Me}$ & 90 & - \\
$\mathbf{8 f}$ & $L$-Trp & $\mathrm{Z}$ & $\mathrm{CH}_{2}-\mathrm{Indol}_{-}-\mathrm{yl}$ & 70 & - \\
$\mathbf{8 g}$ & $D$-Ala & $\mathrm{Z}$ & $\mathrm{CH}_{3}$ & 69 & - \\
\hline
\end{tabular}

${ }^{a}$ Isolated yield. ${ }^{b}$ Yields obtained in refluxing $\mathrm{CH}_{3} \mathrm{CN} .{ }^{c} \mathrm{Lit}^{10}$ Boc-Protected $\mathrm{N}$-carboxyanhydride with 7. ${ }^{d}$ Lit. ${ }^{2 b} \mathrm{EDCl}$, DMAP with Cbz-Ala-OH or Cbz-Val-OH.

\section{Preparation of non-chiral peptidyl phosphorus ylides 10, 11, 12}

Reactions of non-chiral compounds 10-12 were carried out under the optimized microwave condition $\left(60{ }^{\circ} \mathrm{C}, 120 \mathrm{~W}, \mathrm{CH}_{3} \mathrm{CN}, 10\right.$ min.). Reaction of Cbz-Gly-Bt (9a) with 7 produced compound $\mathbf{1 0}$ in $80 \%$ yield. $N$-methylated Gly-Bt (Cbz-Sar-Bt, $\mathbf{9 b}$ ) reacted with $\mathbf{7}$ to give $\mathbf{1 1}$ in $89 \%$ yield. When Cbz-Aib-Bt (9c) was used under the optimized conditions, compound $\mathbf{1 2}$ was obtained in 3\% yield. Extension of the reaction time resulted in decomposition of $\mathbf{9 c}$ and no improvement of the yield was observed. Apparently, the formation of $\mathbf{1 2}$ was inhibited by steric hindrance from the two methyl groups at the $\alpha$-position.
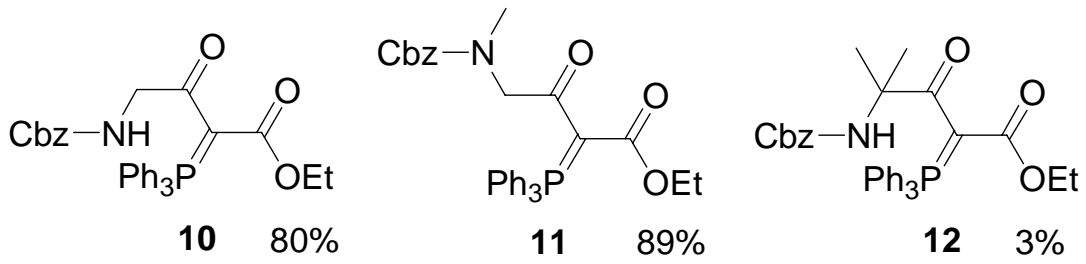

\section{Scheme 3}

\section{Preparation of diastereomeric peptidyl phosphorus ylides}

Synthesis of 15 ( $L L$-config.) and $\mathbf{1 6}$ ( $D L$-config.) was performed to test retention of the original chirality during the formation of acylphosphorus ylides. Compound $\mathbf{1 4}$ was prepared by following a reported procedure ${ }^{6}$ for a similar compound prepared with valine benzyl ester; a coupling of $L$-phenylalanine methyl ester with $\alpha$-bromoacetic acid in the presence of DCC/DMAP gave $\mathbf{1 3}^{9}$ in $95 \%$ yield. Preparation of $\mathbf{1 4}$ was achieved by a reaction with triphenylphosphine in $\mathrm{THF} / \mathrm{Et}_{2} \mathrm{O}$ (1:3) to give the corresponding phosphonium salt 14. Diastereomeric acylphosphorus ylide 15 was obtained in $61 \%$ yield under microwave conditions $\left(60{ }^{\circ} \mathrm{C}, 120 \mathrm{~W}, \mathrm{CH}_{3} \mathrm{CN}, 10 \mathrm{~min}\right.$.) with Cbz-L-Ala-Bt $(\mathbf{6 b})$ in the presence of 1 equivalent of $\mathrm{Et}_{3} \mathrm{~N}$. Similarly, reaction of 14 with Cbz-D-Ala-Bt (6g) gave diastereomer 16 in 66\% yield. The extent 
of preservation of original chirality was estimated as $>95 \%$ by the ${ }^{1} \mathrm{H}$ NMR spectra of the $(L L)$ and $(D L)$ diastereomers, 15 and 16 respectively. While enantiopure alanine methyl on the $(L L)$ diastereomer (15) showed at $0.99 \mathrm{ppm}$, alanine methyl on the $(D L)$ diastereomer (16) showed at $0.86 \mathrm{ppm}$. Additionally the ${ }^{13} \mathrm{C}$ NMR spectra of the two diastereomers showed a broadening of some signals, and a complex series of signals in the aromatic region, especially between 131.5 to $132.2 \mathrm{ppm}$. Upon a heated ${ }^{13} \mathrm{C} \mathrm{NMR}$ experiment, to $60{ }^{\circ} \mathrm{C}$, of the $(D L)$ diastereomer (16), sharpening of the signals occurred and the complex signals separated to show two sets of doublets. In a ${ }^{31} \mathrm{P}$ NMR experiment, two broad singlets at room temperature merged at $60{ }^{\circ} \mathrm{C}$ to form one sharp singlet, indicating the presence of two rotomeric forms. These results confirmed the preservation of chirality.<smiles>COC(=O)C(N)Cc1ccccc1</smiles>
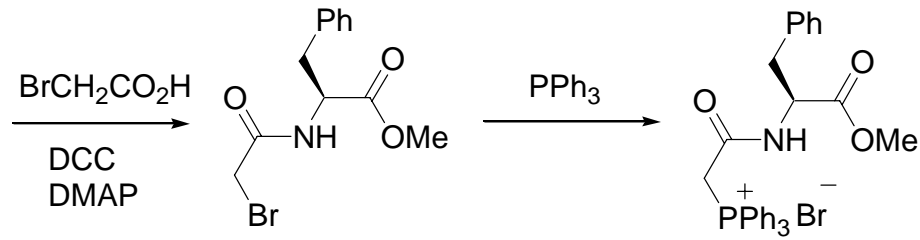

$1395 \%$

$1481 \%$

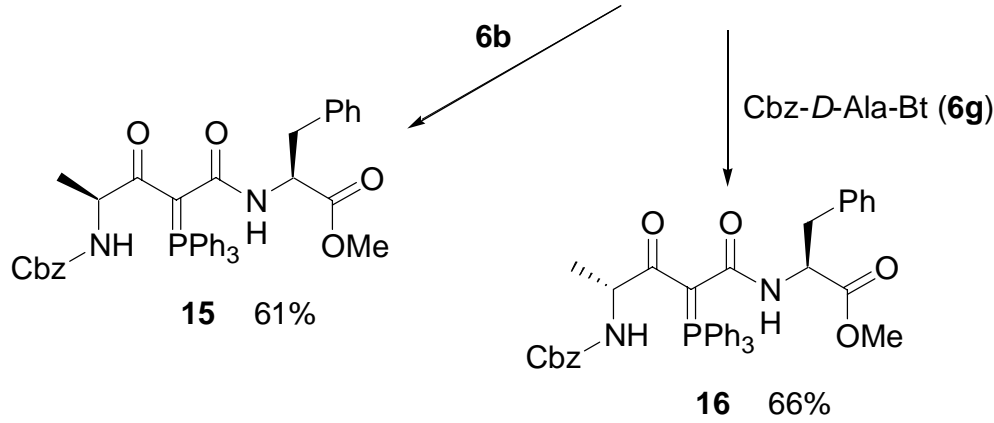

\section{Scheme 4}

\section{Conclusions}

In conclusion, the preparation of peptidyl phosphorus ylides $(\mathbf{8 a}-\mathbf{g}, \mathbf{1 0}, \mathbf{1 1}, \mathbf{1 2}, \mathbf{1 5}$, and 16) utilizing $N$-(Boc- or Cbz- $\alpha$-aminoacyl)benzotriazoles $(\mathbf{6 a}-\mathbf{g}, \mathbf{9 a}-\mathbf{c})$ converted from the corresponding $\mathrm{N}$-protected amino acids was demonstrated under microwave irradiation without base. These results show an application of versatile benzotriazole methodology offering fast and effective synthesis of peptidyl phosphorus ylides using advantageous microwave heating. This procedure utilizes versatile $N$-protected ( $\alpha$-aminoacyl)benzotriazoles to avoid the use of base plus microwave irradiation to reduce reaction times. 


\section{Experimental Section}

General Procedures. Melting points were determined on a capillary point apparatus equipped with a digital thermometer. NMR spectra were recorded in $\mathrm{CDCl}_{3}$ with TMS for ${ }^{1} \mathrm{H}(300 \mathrm{MHz})$ and ${ }^{13} \mathrm{C}(75 \mathrm{MHz})$ as the internal reference, unless otherwise specified. $\mathrm{N}$-Boc- and Cbz-Amino acids purchased from Fluka and amino acids purchased from Acros, were used without further purification. Acetonitrile was purchased from Aldrich, and used without distillation. Microwave heating was carried out with a single mode cavity Discover Microwave Synthesizer (CEM Corporation, NC), producing continuous irradiation at $2455 \mathrm{MHz}$.

General procedure for the preparation of N-protected (aminoacyl)benzotriazoles Compound 6a (Boc protecting group), ${ }^{8 \mathrm{a}} \mathbf{6 b}-\mathbf{f}$ (Cbz protecting group), ${ }^{8 \mathrm{~d}, 8 \mathrm{e}}$ and $\mathbf{9 a}-\mathbf{c}^{8 \mathrm{~d}}$ were prepared by previously reported procedures. Compound $6 \mathbf{6 e}, \mathbf{6 g}$, and $9 \mathbf{a}-\mathbf{c}$ are all novel, and details of their properties and NMR data are described below.

Methyl (3S)-4-(1H-1,2,3-benzotriazol-1-yl)-3-\{[(benzyloxy)carbonyl]amino\}-4-oxobutanoate (Cbz-Asp(OMe)-Bt, 6e). Colorless needles (from chloroform/hexane), 86\% yield, mp 72-74 ${ }^{\circ} \mathrm{C}$. $[\alpha]^{23}{ }_{\mathrm{D}}=-23.4\left(c 1.75, \mathrm{CH}_{2} \mathrm{Cl}_{2}\right) .{ }^{1} \mathrm{H}$ NMR $\delta 3.23(\mathrm{dd}, J=16.6,4.8 \mathrm{~Hz}, 1 \mathrm{H}), 3.38(\mathrm{dd}, J=16.6$, $4.8 \mathrm{~Hz}, 1 \mathrm{H}), 3.65$ (s, 3H), 5.14 (s, 2H), 5.90-5.97 (m, 1H), 6.11 (br s, 1H), 7.35 (br s, 5H), 7.51-7.56 (m, 1H), 7.65-7.71 (m, 1H), $8.13(\mathrm{~d}, J=8.2 \mathrm{~Hz}, 1 \mathrm{H}), 8.27(\mathrm{~d}, J=8.1 \mathrm{~Hz}, 1 \mathrm{H}) .{ }^{13} \mathrm{C}$ NMR $\delta 37.2,51.7,52.2,67.4,114.4,120.3,126.6,128.1,128.2,128.5,130.9,131.2,135.9$, 145.9, 155.7, 169.2, 170.4. Anal. Calcd for $\mathrm{C}_{19} \mathrm{H}_{18} \mathrm{~N}_{4} \mathrm{O}_{5}$ : C, 59.68; H, 4.74; N, 14.65. Found: $\mathrm{C}$, $59.76 ; \mathrm{H}, 4.66 ; \mathrm{N}, 14.58$.

Benzyl $N$-[(1R)-2-(1H-1,2,3-benzotriazol-1-yl)-1-methyl-2-oxoethyl]carbamate (Cbz-D-AlaBt, 6g). White crystals (from ethyl acetate/hexanes), $85 \%$ yield, mp $94-96{ }^{\circ} \mathrm{C} .[\alpha]^{23} \mathrm{D}=+80.2(\mathrm{c}$ 2.08, $\left.\mathrm{CH}_{2} \mathrm{Cl}_{2}\right) .{ }^{1} \mathrm{H}$ NMR $\delta 1.69(\mathrm{~d}, J=7.0 \mathrm{~Hz}, 3 \mathrm{H}), 5.11(\mathrm{~d}, J=12.2 \mathrm{~Hz}, 1 \mathrm{H}$, A part of AB system), 5.17 (d, $J=12.2 \mathrm{~Hz}, 1 \mathrm{H}, \mathrm{B}$ part of AB system), $5.65(\mathrm{~d}, J=6.9 \mathrm{~Hz}, 1 \mathrm{H}), 5.81$ (quintet, $J=7.1 \mathrm{~Hz}, 1 \mathrm{H}), 7.10-7.45(\mathrm{~m}, 5 \mathrm{H}), 7.50-7.56(\mathrm{~m}, 1 \mathrm{H}), 7.64-7.70(\mathrm{~m}, 1 \mathrm{H}), 8.14(\mathrm{~d}, J=8.2 \mathrm{~Hz}$, $1 \mathrm{H}), 8.26(\mathrm{~d}, J=8.2 \mathrm{~Hz}, 1 \mathrm{H}) .{ }^{13} \mathrm{C}$ NMR $\delta 18.9,50.5,67.1,114.3,120.3,126.4,128.1(2 \mathrm{C})$, 128.4, 130.6, 131.1, 136.0, 145.9, 155.6, 172.2. Anal. Calcd for $\mathrm{C}_{17} \mathrm{H}_{16} \mathrm{~N}_{4} \mathrm{O}_{3}$ : C, 62.95; H, 4.97; N, 17.27. Found: C, 62.82; H, 4.97; N, 17.25.

Benzyl $N$-[2-(1H-1,2,3-benzotriazol-1-yl)-2-oxoethyl]carbamate (Cbz-Gly-Bt, 9a). White microcrystals (from chloroform/hexane), 98\% yield, mp 106-108 ${ }^{\circ} \mathrm{C} .{ }^{1} \mathrm{H}$ NMR $\delta 5.10(\mathrm{~d}, J=5.7$ $\mathrm{Hz}, 1 \mathrm{H}), 5.20(\mathrm{~s}, 2 \mathrm{H}), 5.55(\mathrm{~s}, 1 \mathrm{H}), 7.35-7.39(\mathrm{~m}, 5 \mathrm{H}), 7.51-7.56(\mathrm{~m}, 1 \mathrm{H}), 7.66-7.71(\mathrm{~m}, 1 \mathrm{H})$, $8.15(\mathrm{~d}, J=8.2 \mathrm{~Hz}, 1 \mathrm{H}), 8.25(\mathrm{~d}, J=8.4 \mathrm{~Hz}, 1 \mathrm{H}) .{ }^{13} \mathrm{C}$ NMR $\delta 45.0,67.7,114.3,120.6,126.8$, 128.4, 128.5, 128.8, 131.1, 136.2.146.2, 156.7, 168.6. Anal. Calcd for $\mathrm{C}_{16} \mathrm{H}_{14} \mathrm{~N}_{4} \mathrm{O}_{3}: \mathrm{C}, 61.93 ; \mathrm{H}$, 4.55; N, 18.06. Found: C, 61.98; H, 4.57; N, 17.99.

Benzyl $N$-[2-(1H-1,2,3-benzotriazol-1-yl)-2-oxoethyl]- $N$-methylcarbamate (Cbz-Sar-Bt, 9b). (two rotomeric forms) Colorless crystals (from ethyl acetate/hexane), $84 \%$ yield, mp $45-46^{\circ} \mathrm{C}$. ${ }^{1} \mathrm{H}$ NMR $\delta 3.17(\mathrm{~s}, 3 \mathrm{H}), 5.12(\mathrm{~s}, 1 \mathrm{H}), 5.15(\mathrm{~s}, 1 \mathrm{H}), 5.17(\mathrm{~s}, 1 \mathrm{H}), 5.23(\mathrm{~s}, 1 \mathrm{H}), 7.20-7.26(\mathrm{~m}, 2 \mathrm{H})$, 7.34-7.44, (m, 3H), 7.51-7.57 (m, 2H), 7.65-7.72 (m, 2H), 8.13-8.15 (m, 1H), 8.23-8.28 
(m, 1H). ${ }^{13} \mathrm{C}$ NMR $\delta 35.8,36.3,52.4,52.8,67.6,67.8,114.1,120.3,126.4,126.5,127.8,127.9$, 128.0, 128.1, 128.4, 128.5, 130.7, 130.8, 131.0, 136.2, 136.4, 145.9, 156.1, 156.9, 167.8, 167.9. Anal. Calcd for $\mathrm{C}_{17} \mathrm{H}_{16} \mathrm{~N}_{4} \mathrm{O}_{3}$ : C, 62.95; H, 4.97; N, 17.27. Found: C, 62.82; H, 4.99; N, 17.30 .

Benzyl $\mathrm{N}$-[2-(1H-1,2,3-benzotriazol-1-yl)-1,1-dimethyl-2-oxoethyl]carbamate (Cbz-Aib-Bt, 9c). Colorless needles (from chloroform/hexane), 80\% yield, mp 98-100 ${ }^{\circ} \mathrm{C} .{ }^{1} \mathrm{H}$ NMR $\delta 1.88$ (s, $6 \mathrm{H}), 4.90$ (s, 2H), 5.77 (br s, 1H), 7.11-7.20 (m, 5H), 7.47-7.53 (m, 1H), 7.62-7.67 (m, 1H), $8.09(\mathrm{~d}, J=8.2 \mathrm{~Hz}, 1 \mathrm{H}), 8.29(\mathrm{~d}, J=8.2 \mathrm{~Hz}, 1 \mathrm{H}) .{ }^{13} \mathrm{C} \mathrm{NMR} \delta 26.0,58.9,66.8,115.0,119.9$, 126.0, 127.8, 128.0, 128.3, 130.5, 131.9, 135.9, 144.8, 155.3, 172.6. Anal. Calcd for $\mathrm{C}_{18} \mathrm{H}_{18} \mathrm{~N}_{4} \mathrm{O}_{3}$ : C, 63.89; H, 5.36; N, 16.56. Found: C, 63.73; H, 5.22; N, 16.55 .

\section{General procedure for the preparation of peptidyl ylides under the microwave irraditation}

A dry $50 \mathrm{~mL}$ round bottom flask with a magnetic stir bar was equipped with a condenser, and the apparatus was charged with a solution of N-protected (aminoacyl)benzotriazole 6 (1.1 mmol) and ethyl (triphenylphosphoranylidene)acetate $7(0.348 \mathrm{~g}, 1.0 \mathrm{mmol})$ in acetonitrile $(1 \mathrm{~mL})$. The flask containing the reaction mixture was exposed to microwave irradiation $(120 \mathrm{~W})$ for $10 \mathrm{~min}$ at a temperature of $60{ }^{\circ} \mathrm{C}$. The reaction mixture was cooled with high-pressure air through an inbuilt system in the instrument until temperature had fallen below $30{ }^{\circ} \mathrm{C}$ (ca. $2 \mathrm{~min}$ ). The reaction mixture was diluted with ethyl acetate, and the organic solution was washed with saturated aqueous $\mathrm{Na}_{2} \mathrm{CO}_{3}$ solution, water, and dried over $\mathrm{MgSO}_{4}$ to give a crude product, which was purified by column chromatography on silica gel with hexane/ethyl acetate (1:1).

\section{Procedure for the preparation of $8 \mathrm{~b}, 8 \mathrm{~d}$ under the conventional heating}

A dry $50 \mathrm{~mL}$ round bottom flask with a stir bar was attached to a condenser, and the apparatus was charged with a solution of N-protected (aminoacyl)benzotriazole 6 (1.1 mmol) and ethyl (triphenyl phosphoranylidene)acetate $7(0.348 \mathrm{~g}, 1.0 \mathrm{mmol})$ in acetonitrile $(15 \mathrm{~mL})$. The reaction mixture was heated in an oil bath at $70-80{ }^{\circ} \mathrm{C}$ for about 12 hours until the starting materials were completely consumed (Monitored by TLC, hexanes:ethyl acetate $=1: 1$ ). After concentration under reduced pressure, the residue was diluted with ethyl acetate, and the organic solution was washed with saturated $\mathrm{Na}_{2} \mathrm{CO}_{3}$ solution, water, and dried over $\mathrm{MgSO}_{4}$ to give a crude product, which was purified by column chromatography on silica gel with hexane/ethyl acetate $(1: 1)$.

Ethyl (S)-4-tert-butoxycarbonylamino-3-oxo-2-(triphenylphosphanylidene)pentanoate (8a). Colorless microcrystals (from ethyl acetate/hexanes), 65\% yield, mp 153-155 ${ }^{\circ} \mathrm{C}$ (lit. ${ }^{10} \mathrm{mp}$ $\left.153-155^{\circ} \mathrm{C}\right) .[\alpha]^{23}{ }_{\mathrm{D}}=+0.2\left(c 1.67, \mathrm{CH}_{2} \mathrm{Cl}_{2}\right) .{ }^{1} \mathrm{H}$ NMR $\delta 0.75(\mathrm{t}, J=7.0 \mathrm{~Hz}, 3 \mathrm{H}), 1.38(\mathrm{~s}, 9 \mathrm{H})$, $1.43(\mathrm{~d}, J=6.3 \mathrm{~Hz}, 3 \mathrm{H}), 3.65-3.95(\mathrm{~m}, 2 \mathrm{H}), 5.38-5.51(\mathrm{~m}, 2 \mathrm{H}), 7.44-7.68(\mathrm{~m}, 15 \mathrm{H}) .{ }^{13} \mathrm{C}$ NMR $\delta 13.7,20.1,28.3,51.9\left(J_{\mathrm{CP}}=8.0 \mathrm{~Hz}\right), 58.6,68.9\left(J_{\mathrm{CP}}=110.5 \mathrm{~Hz}\right), 78.3,126.1\left(J_{\mathrm{CP}}=93.3 \mathrm{~Hz}\right)$, $128.5\left(J_{\mathrm{CP}}=12.6 \mathrm{~Hz}\right), 131.7,133.0\left(J_{\mathrm{CP}}=9.7 \mathrm{~Hz}\right), 155.2,166.7\left(J_{\mathrm{CP}}=15.5 \mathrm{~Hz}\right), 195.5$.

Ethyl (4S)-4-\{[(benzyloxy)carbonyl]amino\}-3-oxo-2-(triphenylphosphanylidene)pentanoate (8b). Colorless microcrystals (from chloroform/hexane), 86\% yield, mp 140-142 ${ }^{\circ} \mathrm{C}$ (lit. $^{2 \mathrm{~b}}$ $\left.140-142^{\circ} \mathrm{C}\right) .[\alpha]^{23}{ }_{\mathrm{D}}=+25.4\left(c 1.58, \mathrm{CH}_{2} \mathrm{Cl}_{2}\right)\left(\right.$ lit. $\left.^{2 \mathrm{~b}}[\alpha]^{20}{ }_{\mathrm{D}}=+20.3\left[c 1.0005, \mathrm{CH}_{2} \mathrm{Cl}_{2}\right]\right) .{ }^{1} \mathrm{H} \mathrm{NMR}$ $\delta 0.75(\mathrm{t}, J=7.0 \mathrm{~Hz}, 3 \mathrm{H}), 1.47(\mathrm{~d}, J=6.6 \mathrm{~Hz}, 3 \mathrm{H}), 3.69-3.82(\mathrm{~m}, 2 \mathrm{H}), 5.06(\mathrm{~s}, 2 \mathrm{H}), 5.49$ 
(quintet, $J=7.1 \mathrm{~Hz}, 1 \mathrm{H}), 5.85(\mathrm{~d}, J=7.6 \mathrm{~Hz}, 1 \mathrm{H}), 7.27-7.68(\mathrm{~m}, 20 \mathrm{H}) .{ }^{13} \mathrm{C} \mathrm{NMR} \delta 13.7,20.4$, $52.4\left(J_{\mathrm{CP}}=8.6 \mathrm{~Hz}\right), 58.6,65.9,68.8\left(J_{\mathrm{CP}}=111.1 \mathrm{~Hz}\right), 126.0\left(J_{\mathrm{CP}}=93.3 \mathrm{~Hz}\right), 127.6(3 \mathrm{C}), 128.2$, $128.5\left(J_{\mathrm{CP}}=12.6 \mathrm{~Hz}\right), 131.8\left(J_{\mathrm{CP}}=2.9 \mathrm{~Hz}\right), 133.0\left(J_{\mathrm{CP}}=9.7 \mathrm{~Hz}\right), 137.1,155.4,166.7\left(J_{\mathrm{CP}}=14.3\right.$ $\mathrm{Hz})$, 194.7. Anal. Calcd for $\mathrm{C}_{33} \mathrm{H}_{32} \mathrm{NO}_{5} \mathrm{P}: \mathrm{C}, 71.60 ; \mathrm{H}, 5.83 ; \mathrm{N}, 2.53$. Found: C, 71.39; H, 5.78; N, 2.40 .

Ethyl (4S)-4-\{[benzyloxy)carbonyl]amino-5-methyl-3-oxo-2-(triphenylphosphoranylidene) hexanoate (8c). Colorless crystals (from ethyl acetate/hexanes), $88 \%$ yield, mp $88-90{ }^{\circ} \mathrm{C}$ (lit. ${ }^{2 b}$ $\left.88-91{ }^{\circ} \mathrm{C}\right) .[\alpha]^{23}{ }_{\mathrm{D}}=+28.0\left(c 1.66, \mathrm{CH}_{2} \mathrm{Cl}_{2}\right) .{ }^{1} \mathrm{H}$ NMR $\delta 0.68(\mathrm{~d}, J=7.1 \mathrm{~Hz}, 3 \mathrm{H}), 0.73(\mathrm{~d}, J=6.9$ $\mathrm{Hz}, 3 \mathrm{H}), 1.09(\mathrm{~d}, J=6.7 \mathrm{~Hz}, 3 \mathrm{H}), 2.42-2.45(\mathrm{~m}, 1 \mathrm{H}), 3.68-3.85(\mathrm{~m}, 2 \mathrm{H}), 5.06(\mathrm{~s}, 2 \mathrm{H})$, 5.52-5.56 (m, 1H), $5.68(\mathrm{~d}, J=8.9 \mathrm{~Hz}, 7.39-7.20(\mathrm{~m}, 5 \mathrm{H}), 7.51-7.40(\mathrm{~m}, 10 \mathrm{H}), 7.80-7.63(\mathrm{~m}$, 5H). ${ }^{13} \mathrm{C}$ NMR $\delta 13.8,15.9,20.7,32.3,58.6,60.4(J \mathrm{cp}=8.5 \mathrm{~Hz}), 66.0,69.8(J \mathrm{cp}=111.0 \mathrm{~Hz})$, $126.0(\mathrm{Jcp}=93.9 \mathrm{~Hz}), 127.6(3 \mathrm{C}), 128.2,128.5(\mathrm{Jcp}=12.6 \mathrm{~Hz}), 131.8(\mathrm{Jcp}=<2 \mathrm{~Hz}), 133.0(\mathrm{Jcp}$ $=9.7 \mathrm{~Hz}), 137.1,156.6,166.8(\mathrm{Jcp}=14.2 \mathrm{~Hz})$.

Ethyl (4S)-4-\{[(benzyloxy)carbonyl]amino\}-3-oxo-5-phenyl-2-(triphenylphosphanylidene) pentanoate (8d). Colorless foam, 79\% yield, mp 51-53 ${ }^{\circ} \mathrm{C}$. $[\alpha]^{23}{ }_{\mathrm{D}}=+0.58\left(\mathrm{c} 1.66, \mathrm{CH}_{2} \mathrm{Cl}_{2}\right) .{ }^{1} \mathrm{H}$ NMR $\delta 0.71(\mathrm{t}, J=7.1 \mathrm{~Hz}, 3 \mathrm{H}), 2.83(\mathrm{dd}, J=13.2,7.7 \mathrm{~Hz}, 1 \mathrm{H}), 3.40(\mathrm{dd}, J=13.2,4.4 \mathrm{~Hz}, 1 \mathrm{H})$, $3.70-3.85(\mathrm{~m}, 2 \mathrm{H}), 4.95(\mathrm{~d}, J=12.8 \mathrm{~Hz}, 1 \mathrm{H}, \mathrm{A}$ part of AB system), 5.02 (d, $J=12.8 \mathrm{~Hz}, 1 \mathrm{H}, \mathrm{B}$ part of AB system), $5.58(\mathrm{~d}, J=8.9 \mathrm{~Hz}, 1 \mathrm{H}), 5.80-5.87(\mathrm{~m}, 1 \mathrm{H}), 7.16-7.32(\mathrm{~m}, 10 \mathrm{H}), 7.41-7.47$ $(\mathrm{m}, 5 \mathrm{H}), 7.53-7.66(\mathrm{~m}, 10 \mathrm{H}) .{ }^{13} \mathrm{C} \mathrm{NMR} \delta 13.7,39.8,56.8\left(J_{\mathrm{CP}}=8.6 \mathrm{~Hz}\right), 58.7,65.9,70.1\left(J_{\mathrm{CP}}=\right.$ $108.8 \mathrm{~Hz}), 125.9\left(J_{\mathrm{CP}}=93.9 \mathrm{~Hz}\right) 126.0,127.5,127.9,128.2,128.5\left(J_{\mathrm{CP}}=12.6 \mathrm{~Hz}\right), 129.7,131.7$ $\left(J_{\mathrm{CP}}=2.9 \mathrm{~Hz}\right) 133.1\left(J_{\mathrm{CP}}=9.7 \mathrm{~Hz}\right), 137.1,138.0,155.7,166.9\left(J_{\mathrm{CP}}=14.3 \mathrm{~Hz}\right), 193.5$. Anal. Calcd for $\mathrm{C}_{39} \mathrm{H}_{36} \mathrm{NO}_{5} \mathrm{P}: \mathrm{C}, 74.39 ; \mathrm{H}, 5.76 ; \mathrm{N}, 2.22$. Found: C, 74.10; H, 5.83; N, 2.58 .

1-Ethyl 6-methyl (4S)-4-\{[(benzyloxy)carbonyl]amino\}-3-oxo-2-(triphenyl phosphanylidene)hexanedioate (8e). Colorless microcrystals (from ethyl acetate/hexanes), 90\% yield, mp $116-118{ }^{\circ} \mathrm{C} .[\alpha]^{23} \mathrm{D}=+0.84\left(c 1.91, \mathrm{CH}_{2} \mathrm{Cl}_{2}\right) .{ }^{1} \mathrm{H} \mathrm{NMR} \delta 0.72(\mathrm{t}, J=6.9 \mathrm{~Hz}, 3 \mathrm{H}), 2.82(\mathrm{dd}, J=$ $14.3,6.7 \mathrm{~Hz}, 1 \mathrm{H}), 3.09$ (dd, $J=14.3,3.4 \mathrm{~Hz}, 1 \mathrm{H}), 3.56(\mathrm{~s}, 3 \mathrm{H}), 3.69-3.85$ (m, 2H), $5.06(\mathrm{~s}, 2 \mathrm{H})$, $5.76-5.81(\mathrm{~m}, 1 \mathrm{H}), 5.91(\mathrm{~d}, J=8.1 \mathrm{~Hz}, 1 \mathrm{H}), 7.22-7.72(\mathrm{~m}, 20 \mathrm{H}) .{ }^{13} \mathrm{C}$ NMR $\delta 13.6,38.6,51.5$, $53.6\left(J_{\mathrm{CP}}=9.2 \mathrm{~Hz}\right), 58.8,66.1,69.3\left(J_{\mathrm{CP}}=109.4 \mathrm{~Hz}\right), 125.6\left(J_{\mathrm{CP}}=93.9 \mathrm{~Hz}\right), 127.5,127.6,128.2$, $128.5\left(J_{\mathrm{CP}}=12.6 \mathrm{~Hz}\right), 131.8\left(J_{\mathrm{CP}}=2.9 \mathrm{~Hz}\right), 133.1\left(J_{\mathrm{CP}}=9.7 \mathrm{~Hz}\right), 136.9,155.6,166.7\left(J_{\mathrm{CP}}=\right.$ 14.3 Hz), 171.5, 191.8. Anal. Calcd for $\mathrm{C}_{35} \mathrm{H}_{34} \mathrm{NO}_{7} \mathrm{P}: \mathrm{C}, 68.73 ; \mathrm{H}, 5.60 ; \mathrm{N}, 2.29$. Found: C, 68.66; H, 5.65; N, 2.22 .

Ethyl (S)-4-benzyloxycarbonylamino-5-(1H-indol-3-yl)-3-oxo-2-(triphenyl-phosphanylidene)pentanoate (8f). White microcrystals (from chloroform/hexanes), $71 \%$ yield, $\mathrm{mp}$ $88-90{ }^{\circ} \mathrm{C} .[\alpha]^{23}{ }_{\mathrm{D}}=+40.0\left(c 1.67, \mathrm{CH}_{2} \mathrm{Cl}_{2}\right) .{ }^{1} \mathrm{H} \mathrm{NMR} \delta 0.72(\mathrm{t}, J=7.0 \mathrm{~Hz}, 3 \mathrm{H}), 3.26(\mathrm{dd}, J=$ 14.7, $6.9 \mathrm{~Hz}, 1 \mathrm{H}), 3.51(\mathrm{dd}, J=14.7,4.5 \mathrm{~Hz}, 1 \mathrm{H}), 3.68-3.83(\mathrm{~m}, 2 \mathrm{H}), 4.97(\mathrm{~s}, 2 \mathrm{H}), 5.70-5.80$ $(\mathrm{m}, 1 \mathrm{H}), 5.80-5.91(\mathrm{~m}, 1 \mathrm{H}), 6.91(\mathrm{~s}, 1 \mathrm{H}), 7.00-7.40(\mathrm{~m}, 15 \mathrm{H}), 7.40-7.60(\mathrm{~m}, 9 \mathrm{H}), 7.71(\mathrm{~d}, J=$ $7.6 \mathrm{~Hz}, 1 \mathrm{H}), 7.90(\mathrm{~s}, 1 \mathrm{H}) .{ }^{13} \mathrm{C} \mathrm{NMR} \delta 13.7,28.8,56.5\left(J_{\mathrm{CP}}=8.6 \mathrm{~Hz}\right), 58.8,68.3\left(J_{\mathrm{CP}}=96.3 \mathrm{~Hz}\right)$, $110.9,111.5,119.0,121.3,122.9,125.9\left(J_{\mathrm{CP}}=93.3 \mathrm{~Hz}\right), 127.6,127.9,128.2,128.5\left(J_{\mathrm{CP}}=\right.$ $12.6 \mathrm{~Hz}), 131.6,132.0,132.1,133.0\left(J_{\mathrm{CP}}=9.7 \mathrm{~Hz}\right), 135.9,137.0,155.8,166.9\left(J_{\mathrm{CP}}=13.7 \mathrm{~Hz}\right)$, 
193.9. Anal. Calcd for $\mathrm{C}_{41} \mathrm{H}_{37} \mathrm{~N}_{2} \mathrm{O}_{5} \mathrm{P}$ : C, 73.64; H, 5.58; N, 4.19. Found: C, 73.07; H, 5.58; N, 4.16. HRMS $m / z$ Calcd for $\mathrm{C}_{41} \mathrm{H}_{37} \mathrm{~N}_{2} \mathrm{O}_{5} \mathrm{P} 669.2513\left(\mathrm{M}+\mathrm{H}^{+}\right)$, Found 5569.2523.

Ethyl (R)-4-benzyloxycarbonylamino-3-oxo-2-(triphenylphosphanylidene)-pentanoate (8g). Colorless crystals (from ethyl acetate/hexane), 69\% yield, mp $135-137^{\circ} \mathrm{C}$. $[\alpha]^{23}{ }_{\mathrm{D}}=-17.5$ (c 2.08, $\left.\mathrm{CH}_{2} \mathrm{Cl}_{2}\right) .{ }^{1} \mathrm{H}$ NMR $\delta 0.75(\mathrm{t}, J=7.1 \mathrm{~Hz}, 3 \mathrm{H}), 1.48(\mathrm{~d}, J=7.1 \mathrm{~Hz}, 3 \mathrm{H}), 3.66-3.88(\mathrm{~m}, 2 \mathrm{H})$, $5.06(\mathrm{~s}, 2 \mathrm{H}), 5.50$ (quintet, $J=6.7 \mathrm{~Hz}, 1 \mathrm{H}), 5.86(\mathrm{~d}, J=7.7 \mathrm{~Hz}, 1 \mathrm{H}), 7.26-7.68(\mathrm{~m}, 20 \mathrm{H}) .{ }^{13} \mathrm{C}$ NMR $\delta 13.7,20.3,52.4\left(J_{\mathrm{CP}}=8.6 \mathrm{~Hz}\right), 58.6,65.8,68.8\left(J_{\mathrm{CP}}=110.5 \mathrm{~Hz}\right), 125.8\left(J_{\mathrm{CP}}=93.9 \mathrm{~Hz}\right)$, 127.5, 127.6, 128.2, $128.5\left(J_{\mathrm{CP}}=12.6 \mathrm{~Hz}\right), 131.7,131.8\left(J_{\mathrm{CP}}=2.9 \mathrm{~Hz}\right), 132.9\left(J_{\mathrm{CP}}=9.7 \mathrm{~Hz}\right)$, 137.0, 155.4, $166.7\left(J_{\mathrm{CP}}=14.3 \mathrm{~Hz}\right), 194.8$. Anal. Calcd for $\mathrm{C}_{33} \mathrm{H}_{32} \mathrm{NO}_{5} \mathrm{P}: \mathrm{C}, 71.60 ; \mathrm{H}, 5.83 ; \mathrm{N}$, 2.53. Found: C, 71.20; H, 5.89; N, 2.56.

Ethyl 4-benzyloxycarbonylamino-3-oxo-2-(triphenylphosphanylidene)butanoate (10). White microcrystals (from ethyl acetate/hexanes), 80\% yield, mp $134-136{ }^{\circ} \mathrm{C}$ (lit. ${ }^{10} \mathrm{mp} 134-136{ }^{\circ} \mathrm{C}$ ). ${ }^{1} \mathrm{H}$ NMR $\delta 0.75(\mathrm{t}, J=7.0 \mathrm{~Hz}, 3 \mathrm{H}), 3.70-3.82(\mathrm{~m}, 2 \mathrm{H}), 4.60(\mathrm{~d}, J=4.0 \mathrm{~Hz}, 2 \mathrm{H}), 5.06(\mathrm{~s}, 2 \mathrm{H})$, $5.85(\mathrm{~s}, 1 \mathrm{H}), 7.22-7.70(\mathrm{~m}, 20 \mathrm{H}) .{ }^{13} \mathrm{C} \mathrm{NMR} \delta 13.8,49.3\left(J_{\mathrm{CP}}=8.6 \mathrm{~Hz}\right), 58.6,66.1,68.9\left(J_{\mathrm{CP}}=\right.$ $112.8 \mathrm{~Hz}) 125.7\left(J_{\mathrm{CP}}=93.3 \mathrm{~Hz}\right), 127.6,127.7,128.6\left(J_{\mathrm{CP}}=12.6 \mathrm{~Hz}\right), 131.9\left(J_{\mathrm{CP}}=2.9 \mathrm{~Hz}\right), 133.1$ $\left(J_{\mathrm{CP}}=9.7 \mathrm{~Hz}\right), 136.9,156.1,167.3\left(J_{\mathrm{CP}}=14.3 \mathrm{~Hz}\right), 190.3$. Anal. Calcd for $\mathrm{C}_{32} \mathrm{H}_{30} \mathrm{NO}_{5} \mathrm{P}: \mathrm{C}$, 71.23; H, 5.60; N, 2.60. Found: C, 71.11; H, 5.79; N, 2.63.

Ethyl 4-(benzyloxycarbonyl-methyl-amino)-3-oxo-2-(triphenylphosphanylidene)butanoate (11). (two rotomeric forms) White microcrystals (from ethyl acetate/hexanes), 89\% yield, mp 133-135 ${ }^{\circ} \mathrm{C} .{ }^{1} \mathrm{H}$ NMR $\delta 0.64(\mathrm{~d}, J=7.0 \mathrm{~Hz}, 3 \mathrm{H}), 0.70(\mathrm{~d}, J=6.9 \mathrm{~Hz}, 3 \mathrm{H}), 2.83$ (s, 3H), 2.85 $(\mathrm{s}, 3 \mathrm{H}), 3.74$ (quintet, $J=7.1 \mathrm{~Hz}, 2 \mathrm{H}), 4.69(\mathrm{~s}, 2 \mathrm{H}), 5.04(\mathrm{~s}, 1 \mathrm{H}), 5.06(\mathrm{~s}, 1 \mathrm{H}), 7.25-7.70(\mathrm{~m}$, 20H). ${ }^{13} \mathrm{C}$ NMR $\delta 13.5,13.7,35.5,36.1,57.1\left(J_{\mathrm{CP}}=8.6 \mathrm{~Hz}\right), 57.5\left(J_{\mathrm{CP}}=8.0 \mathrm{~Hz}\right), 58.2,66.3$, 66.6, $68.6\left(J_{\mathrm{CP}}=109.9 \mathrm{~Hz}\right), 68.9\left(J_{\mathrm{CP}}=111.7 \mathrm{~Hz}\right), 125.9\left(J_{\mathrm{CP}}=93.3 \mathrm{~Hz}\right), 126.0\left(J_{\mathrm{CP}}=93.3 \mathrm{~Hz}\right)$, $126.9,127.2,127.4,127.5,128.1,128.1,128.3\left(J_{\mathrm{CP}}=12.6 \mathrm{~Hz}\right), 128.3\left(J_{\mathrm{CP}}=12.6 \mathrm{~Hz}\right), 131.5$, $131.5,131.6,131.8,131.9,132.9,133.0,133.1,137.0,137.3,156.6,156.7,167.5$ 167.7, 167.9, $191.1\left(J_{\mathrm{CP}}=3.4 \mathrm{~Hz}\right), 191.6\left(J_{\mathrm{CP}}=3.4 \mathrm{~Hz}\right)$. HRMS $m / z$ Calcd for $\mathrm{C}_{33} \mathrm{H}_{32} \mathrm{NO}_{5} \mathrm{P} 554.2091\left(\mathrm{M}+\mathrm{H}^{+}\right)$, Found 554.2106.

Ethyl 4-benzyloxycarbonylamino-4-methyl-3-oxo-2-(triphenylphosphanylidene)pentanoate (12). White crystals (from ethyl acetate/hexanes), 3\% yield, mp 80-81 ${ }^{\circ} \mathrm{C} .{ }^{1} \mathrm{H} \mathrm{NMR}\left(\mathrm{CDCl}_{3}\right) \delta$ $0.56(\mathrm{t}, J=7.1 \mathrm{~Hz}, 3 \mathrm{H}), 1.63(\mathrm{~s}, 6 \mathrm{H}), 3.58$ (q, $J=7.1 \mathrm{~Hz}, 2 \mathrm{H}), 5.14$ (br s, 2H), 6.79 (s, 1H), 7.25-7.68 (m, 20H). ${ }^{13} \mathrm{C} \mathrm{NMR}\left(\mathrm{CDCl}_{3}\right) \delta 13.5,25.0,58.7,60.1,60.2,65.6,68.9\left(J_{\mathrm{CP}}=109.4\right.$ $\mathrm{Hz}), 127.0\left(J_{\mathrm{CP}}=93.9 \mathrm{~Hz}\right), 127.4,127.5,128.3,128.5\left(J_{\mathrm{CP}}=12.0 \mathrm{~Hz}\right), 131.4\left(J_{\mathrm{CP}}=2.9 \mathrm{~Hz}\right)$, $132.9\left(J_{\mathrm{CP}}=9.7 \mathrm{~Hz}\right), 137.4,155.8,167.2\left(J_{\mathrm{CP}}=13.2 \mathrm{~Hz}\right), 198.2$. HRMS $\mathrm{m} / z$ Calcd for $\mathrm{C}_{34} \mathrm{H}_{34} \mathrm{NO}_{5} \mathrm{P} 568.2247\left(\mathrm{M}+\mathrm{H}^{+}\right)$, Found 568.2269.

[((S)-1-Methoxycarbonyl-2-phenyl-ethylcarbamoyl)-methyl]-triphenylphosphonium bromide (14). White crystal (from dichloromethane/hexanes), $81 \%$ yield, mp $155-157^{\circ} \mathrm{C},[\alpha]^{23}{ }_{\mathrm{D}}=-9.7(c$ 2.08, $\mathrm{CH}_{2} \mathrm{Cl}_{2}$ ). ${ }^{1} \mathrm{H}$ NMR (DMSO- $\left.d_{6}\right) \delta 2.83(\mathrm{dd}, J=13.9,8.8 \mathrm{~Hz}, 1 \mathrm{H}), 2.97(\mathrm{dd}, J=13.9,5.5$ $\mathrm{Hz}, 1 \mathrm{H}), 3.56(\mathrm{~s}, 3 \mathrm{H}), 4.38-4.43(\mathrm{~m}, 1 \mathrm{H}), 5.02-5.09(\mathrm{~m}, 2 \mathrm{H}), 7.13-7.35(\mathrm{~m}, 5 \mathrm{H}), 7.50-7.91(\mathrm{~m}$, $15 \mathrm{H}), 9.07(\mathrm{~d}, J=7.6 \mathrm{~Hz}, 1 \mathrm{H}) .{ }^{13} \mathrm{C}$ NMR $\left(\mathrm{DMSO}-d_{6}\right) \delta 30.6\left(J_{\mathrm{CP}}=57.3 \mathrm{~Hz}\right), 36.5,52.0,54.3$, $118.6\left(J_{\mathrm{CP}}=88.2 \mathrm{~Hz}\right), 126.7,128.3,129.1,129.9\left(J_{\mathrm{CP}}=13.2 \mathrm{~Hz}\right), 133.7\left(J_{\mathrm{CP}}=10.3 \mathrm{~Hz}\right), 134.8$, 
136.5, $163.0\left(J_{\mathrm{CP}}=4.6 \mathrm{~Hz}\right)$, 170.9. Anal. Calcd for $\mathrm{C}_{30} \mathrm{H}_{29} \mathrm{BrNO}_{3} \mathrm{P}: \mathrm{C}, 64.06 ; \mathrm{H}, 5.20 ; \mathrm{N}, 2.49$. Found: C, 63.76; H, 5.18; N, 2.41.

Methyl-(S)-2-[(S)-4-benzyloxycarbonylamino-3-0xo-2-(triphenylphosphanylidene)pentanoylamino]3-phenyl-propionate (15). (two rotomeric forms) White crystals (from dichloromethane/hexanes), $61 \%$ yield, mp $65-68{ }^{\circ} \mathrm{C},[\alpha]^{23}{ }_{\mathrm{D}}=-20.0\left(c 2.08, \mathrm{CH}_{2} \mathrm{Cl}_{2}\right) .{ }^{1} \mathrm{H} \mathrm{NMR} \delta 0.99(\mathrm{~d}, J=4.8 \mathrm{~Hz}, 3 \mathrm{H})$, $1.87(\mathrm{~s}, 1 \mathrm{H}), 2.99(\mathrm{dd}, J=13.5,8.5 \mathrm{~Hz}, 1 \mathrm{H}), 3.12(\mathrm{dd}, J=13.5,5.2 \mathrm{~Hz}, 1 \mathrm{H}), 3.61(\mathrm{~s}, 3 \mathrm{H}), 4.65-$ $4.72(\mathrm{~m}, 1 \mathrm{H})$ 4.95-5.06 (m, 2H), $5.66($ br s, $1 \mathrm{H}), 7.23-7.70(\mathrm{~m}, 26 \mathrm{H}) .{ }^{13} \mathrm{C} \mathrm{NMR} \delta 20.4,38.1$, 50.5, 51.7, 53.6, 65.9, $72.2\left(J_{\mathrm{CP}}=116.8 \mathrm{~Hz}\right), 126.1\left(\mathrm{~d}, J_{\mathrm{CP}}=93.3 \mathrm{~Hz},\right), 126.3,127.5,127.6$, $128.0,128.1,128.2,128.3,128.5\left(J_{\mathrm{CP}}=12.6 \mathrm{~Hz}\right), 129.2,131.5,131.7,131.9,132.9\left(J_{\mathrm{CP}}=\right.$ 9.7 Hz), 136.6, 137.0, 155.1, 168.5, 172.6, 191.2. Anal. Calcd for $\mathrm{C}_{41} \mathrm{H}_{39} \mathrm{~N}_{2} \mathrm{O}_{6} \mathrm{P}: \mathrm{C}, 71.71 ; \mathrm{H}$, $5.72 ; \mathrm{N}, 4.08$. Found: C, 71.85; H, 5.81; N, 3.75.

Methyl-(S)-2-[(R)-4-benzyloxycarbonylamino-3-oxo-2-(triphenylphosphanylidene)-pentanoylamino]3-phenyl-propionate (16). (two rotomeric forms) White crystals (from dichloromethane/hexanes), $66 \%$ yield, mp 46-48 $\left.{ }^{\circ} \mathrm{C},[\alpha]^{23}{ }_{\mathrm{D}}=+4.4(c) 2.08, \mathrm{CH}_{2} \mathrm{Cl}_{2}\right) .{ }^{1} \mathrm{H}$ NMR $\delta 0.86(\mathrm{~d}, J=6.7 \mathrm{~Hz}, 3 \mathrm{H})$, 1.90 (s, 1H), 2.98 (dd, $J=13.8,7.9 \mathrm{~Hz}, 1 \mathrm{H}), 3.11$ (dd, $J=13.6,5.4 \mathrm{~Hz}, 1 \mathrm{H}), 3.61$ (s, 3H), 4.67$4.74(\mathrm{~m}, 1 \mathrm{H}) 5.03$ (br s, 2H), 5.60 (br s, 1H), 7.15-7.70 (m, 26H). ${ }^{13} \mathrm{C}$ NMR $\delta 20.3,38.1,50.6$, $51.8\left(J_{\mathrm{CP}}=4.6 \mathrm{~Hz}\right), 53.7,66.1,72.8\left(J_{\mathrm{CP}}=119.7 \mathrm{~Hz}\right), 126.5\left(J_{\mathrm{CP}}=93.9 \mathrm{~Hz},\right), 126.5,127.7$, $127.8,128.3,128.4,128.5,128.7\left(J_{\mathrm{CP}}=12.6 \mathrm{~Hz}\right), 129.2,131.7,131.9,131.9,132.0,132.1,133.2$ $\left(J_{\mathrm{CP}}=9.7 \mathrm{~Hz}\right), 136.7,137.1,155.3,169.2,172.8,191.5 .\left[{ }^{13} \mathrm{C} \mathrm{NMR}\left(\mathrm{CDCl}_{3}, 60{ }^{\circ} \mathrm{C}\right.\right.$, aromatic region) $\delta 127.0\left(J_{\mathrm{CP}}=93.7 \mathrm{~Hz},\right), 126.5,127.8,128.3,128.4,128.5\left(J_{\mathrm{CP}}=12.1 \mathrm{~Hz}\right), 128.7\left(J_{\mathrm{CP}}=\right.$ $12.6 \mathrm{~Hz}), 129.3,131.8\left(J_{\mathrm{CP}}=3.3 \mathrm{~Hz}\right), 131.9\left(J_{\mathrm{CP}}=3.0 \mathrm{~Hz}\right), 132.2\left(J_{\mathrm{CP}}=9.8 \mathrm{~Hz}\right), 133.4\left(J_{\mathrm{CP}}=\right.$ 9.8 Hz), 136.7, 137.1.]. Anal. Calcd for $\mathrm{C}_{41} \mathrm{H}_{39} \mathrm{~N}_{2} \mathrm{O}_{6} \mathrm{P}: \mathrm{C}, 71.71 ; \mathrm{H}, 5.72 ; \mathrm{N}, 4.08$. Found: C, 71.34; H, 5.89; N, 3.51 .

\section{Acknowledgements}

We thank Dr. Novruz G. Akhmedov (University of Florida) for helping NMR analysis and giving us useful advice.

\section{References}

1. (a) Ocain, T. D.; Rich, D. H. J. Med. Chem. 1992, 35, 451. (b) Patel, D. V.; Rielly-Gauvin, K.; Ryono, D. E.; Free, C. A.; Smith, S. A.; Petrillo, E. W. Jr. J. Med. Chem. 1993, 36, 2431. (c) Yuan, W.; Munoz, B.; Wong, C.-H. J. Med. Chem. 1993, 36, 211.

2. (a) Abell, A. D.; Heinicke, G. W.; Massy-Westropp, R. A. Synthesis 1985, 764. (b) Aitken, R. A.; Karodia, N.; Massil, T.; Young, R. J. J. Chem. Soc. Perkin Trans 1 2002, 533. (c) Aitken, R. A.; Buchanan, G. M.; Karodia, N.; Massil, T.; Young, R. J. Tetrahedron Lett. 2001, 42, 141. (d) Aitken, R. A.; Al-Awadi, N. A.; Dawson, G.; El-Dusouqi, O. M. E.; 
Farrell, D. M. M.; Kaul, K.; Kumar, A. Tetrahedron 2005, 61, 129. (e) RamaRao, V. V. V. N. S.; Reddy, G. V.; Maitraie, D.; Ravikanth, S.; Yadla, R.; Narsaiah, B.; Rao, P. S. Tetrahedron 2004, 60, 12231.

3. (a) Papanikos, A.; Meldal, M. J. Comb. Chem. 2004, 6, 181. (b) Wasserman, H. H.; Ennis, D. S.; Power, P. L.; Ross, M. J.; Gomes, B. J. Org. Chem. 1993, 58, 4785.

4. Cooke, M. P. Jr. J. Org. Chem. 1982, 47, 4963.

5. (a) Cooke, M. P. Jr.; Burman, D. L. J. Org. Chem. 1982, 47, 4955. (b) Wasserman, H. H.; Ho, W.-B. J. Org. Chem. 1994, 59, 4364.

6. Wasserman, H. H.; Chen, J.-H.; Xia, M. J. Am. Chem. Soc. 1999, 121, 1401.

7. (a) Wasserman, H. H.; Baldino, C. M.; Coats, S. J. J. Org. Chem. 1995, 60, 8231. (b) Wasserman, H. H.; Petersen, A. K. J. Org. Chem. 1997, 62, 8972.

8. (a) Katritzky, A. R.; Wang, M.; Yang, H.; Zhang, S.; Akhmedov, N. G. ARKIVOC 2002, (viii), 134. (b) Katritzky, A. R.; Hoffmann, S.; Suzuki, K. ARKIVOC 2004, (xii), 14. (c) Katritzky, A. R.; Shestopalov, A. A.; Suzuki, K. Synthesis 2004, 1402. (d) Katritzky, A. R.; Suzuki, K., and Singh, S. K. Synthesis 2004, 2645. (e) Katritzky, A. R.; Angrish, P.; Hür, D; Suzuki, K. Synthesis 2004, in press. (f) Katritzky, A. R.; Jiang, R.; Suzuki, K. in preparation. (g) Hayes, B. L. Microwave Synthesis: Chemistry at the Speed of Light, CEM Publishing: Matthews, NC, 2002. (h) Katritzky, A. R.; Singh, S. K. ARKIVOC 2003, (xiii), 68.

9. Van, E.; Fernandez-Suarez, M.; Muñoz, L. Tetrahedron: Asymmetry 2003, 14, 1935.

10. Fehrentz, J.-A.; Genu-Dellac, C.; Amblard, M.; Winternitz, F.; Loffet, A.; Martinez, J. J. Pept. Sci. 1995, 1, 124. 